\title{
Diagnostic dilemma: Metastatic bone malignancy or primary hyperparathyroidism with brown tumor*
}

\author{
Esra Hatipoglu ${ }^{1}$, Ahmet Emre Eskazan ${ }^{2}$, Ozlem Celik ${ }^{1}$, Fatih Kantarci ${ }^{3}$, Pinar Kadioglu ${ }^{1 \#}$ \\ ${ }^{1}$ Division of Endocrinology and Metabolism, Department of Internal Medicine, Cerrahpasa Medical School, University of Istanbul, \\ Istanbul, Turkey \\ ${ }^{2}$ Division of Hematology, Department of Internal Medicine, Cerrahpasa Medical School, University of Istanbul, Istanbul, Turkey \\ ${ }^{3}$ Department of Radiology, Cerrahpasa Medical School, University of Istanbul, Istanbul, Turkey \\ Email: "kadioglup@yahoo.com
}

Received 3 April 2013; revised 3 May 2013; accepted 11 May 2013

Copyright (C) 2013 Esra Hatipoglu et al. This is an open access article distributed under the Creative Commons Attribution License, which permits unrestricted use, distribution, and reproduction in any medium, provided the original work is properly cited.

\begin{abstract}
Multiple osteolytic lesions are usually associated with metastatic involvement of the bone. However metabolic bone diseases should also take their place in differential diagnosis. Here, we describe a primary hyperparathyroidism case with full-blown osteolytic lesions which was diagnosed at first sight with having metastatic bone involvement. PET CT scan and laboratory results excluded a metastatic bone malignancy. Elevated serum calcium of $13.16 \mathrm{mg} / \mathrm{dl}$, decreased serum phoshorus of $1.4 \mathrm{mg} / \mathrm{dl}$ and high intact-PTH level of $1054.7 \mathrm{gg} / \mathrm{ml}$ pointed out primary hyperparathyroidism. Sonographic examination revealed two adenomas of $2.9 \times 3.3 \mathrm{~mm}$ and $3.3 \times 2.7 \mathrm{~mm}$ in the left superior and right inferior parathyroid glands, respectively. Scintigraphy confirmed the presence of adenoma on the left.
\end{abstract}

Keywords: Brown Tumor; Hyperparathyroidism; Metastatic Bone Disease

\section{INTRODUCTION}

Primary hyperparathyroidism (PHPT) is usually unveiled during the search of hypercalcemia etiology. Parathyroid adenoma is the leading cause of PHPT [1]. Skeletal changes in primary hyperparathroidism involve bone resorption, cysts and osteopenia. Another skelatal change, brown tumor is one of the rare consequences of hyperparathroidism. Therefore, brown tumor is not a standard means

\footnotetext{
*Declaration of Interest: The authors declare that there is no conflict of interest that could be perceived as prejudicing the impartiality of the research reported.

Funding: This research did not receive any specific grant from any funding agency in the public commercial, or not-for-profit sector.

\#Corresponding author.
}

of presentation in PHPT as it is not a common cause of osteolytic bone involvement [2]. Herein we report an unusual case of PHPT and brown tumor presented with full-blown osteolytic lesions which were firstly confused with metastatic bone involvement.

\section{CASE REPORT}

A 55-year-old woman presented with early fatigability and persistant left hip and thigh pain. The X-rays taken at another hospital showed multiple osteolytic lesions in the left pelvic area and on bilateral femoral neck. She was referred to our institution with the diagnosis of metastatic bone malignancy.

The patient's medical history was significant for bilateral nephrolithiasis and osteoporosis. The pain had a progressive characteristic, disturbing her during sleep. The physical examination did not reveal a significant pathology. Serial plain radiographies showed previosuly noted multiple osteolytic lesions (Figure 1). Magnetic resonance imaging of the hip revealed low signals on T1-weighted and high-signal on T2-weighted images of multiple osteolytic lesions which were present on the left iliac bone and on bilateral femoral neck (Figure 2). To search for a possible primary tumor PET CT of the whole body was obtained. Only the first metatarsal of the right foot had an increased FDG uptake consistent with an inflammatory process. The hypodense signals of the pelvic bones, bilateral femur and proximal of humerus on CT images (Figure 3) were not accompanied by significant FDG uptake on PET images. Laboratory studies including serum protein electrophoresis, complete blood count and routine biochemical analysis, other than an elevated serum calcium of $13.16 \mathrm{mg} / \mathrm{dl}$ and a decreased serum phoshorus of $1.4 \mathrm{mg} / \mathrm{dl}$, were normal. In the light of the PET CT scan and laboratory results a metastatic 


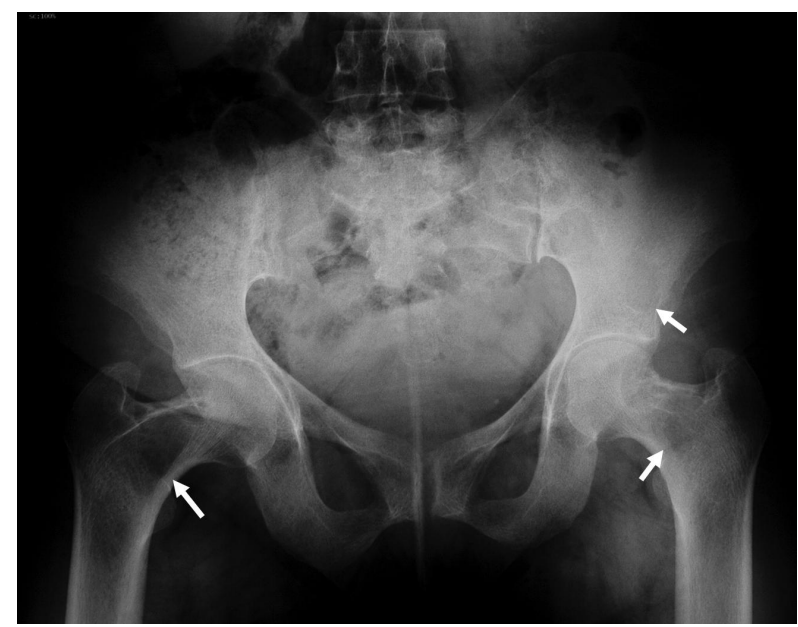

Figure 1. Anteroposterior plain radiography of the pelvis shows multiple radiolucent lytic areas (white arrows).

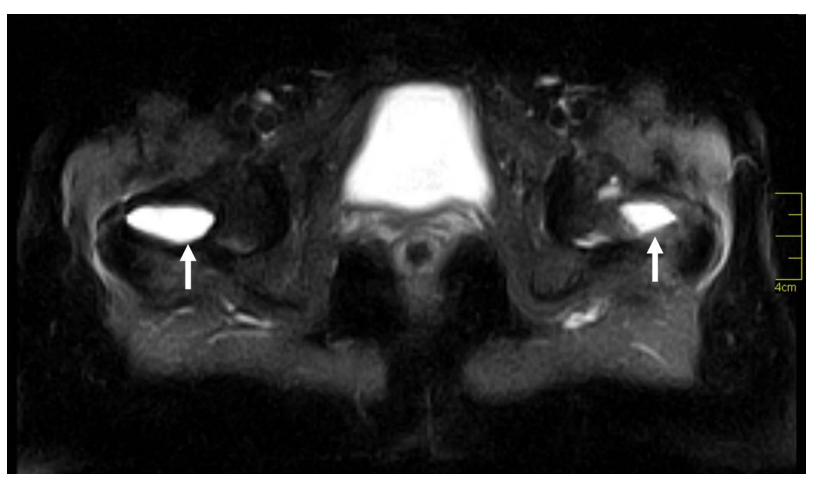

Figure 2. Proton density weighted fat saturated axial MR image at the level of both femoral neck shows well defined multiple cystic (white arrows) lesions.

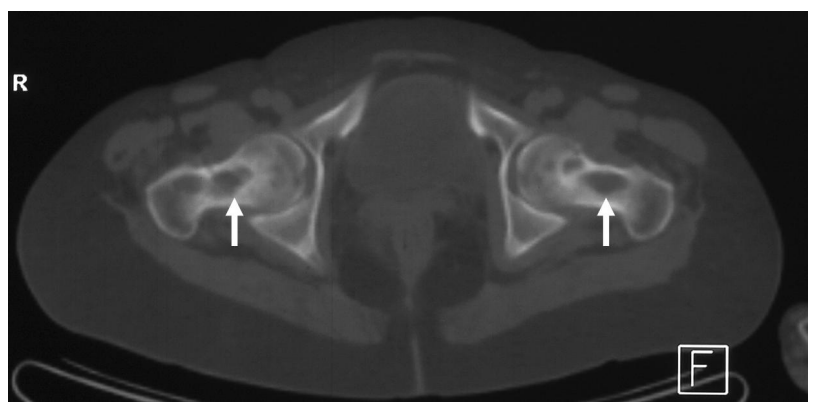

Figure 3. Computed tomography section on axial plane reveals well defined cystic areas (white arrows) at the bilateral femoral neck.

bone malignancy was not of concern anymore. Search for a potential diagnosis of a metabolic bone disease revealed a high intact-PTH level of $1054.7 \mathrm{pg} / \mathrm{ml}$. In order to unveil the cause of hyperparathyroidism sonographic examination was performed and it revealed two adenomas of $2.9 \times 3.3 \mathrm{~mm}$ and $3.3 \times 2.7 \mathrm{~mm}$ in the left superior and right inferior parathyroid glands, respectively. Scintigraphy confirmed the presence of adenoma on the left. Considering the last evidences, brown tumor caused by parathyroid adenoma was the most probable diagnosis.

During her follow-up at in-patient clinic, hypercalcemia of the patient did not improve with intravenous hydration and furosemide. Awaiting the surgical procedure intravenous zolendronic acid infusion was performed. Calcium levels decreased to $11.3 \mathrm{mg} / \mathrm{dl}$ before the operation. After excision of left parathyroid adenoma calcium and PTH levels further decreased until $9.1 \mathrm{mg} / \mathrm{dl}$ and 34 $\mathrm{pg} / \mathrm{ml}$, respectively.

\section{DISCUSSION}

Finding of multiple osteolytic lesions usually is thought to be hall mark of metastatic involvement. Therefore in case of osteolytic bone involvement a tumor survey should be accomplished. Medical history, physical examination, laboratory tests and imaging techniques are the key screening tools for diagnosis of a primary tumor [3-5].

However, metabolic bone diseases should also be taken into consideration. In our patient, presence of multiple osteolytic foci was consistent with metastasis but when medical history, biochemical and radiological analysis were evaluated all together, diagnosis of a malignity became less likely. History of urolithiasis and osteoporosis in conjunction with the marked hypercalcemia, hypophospatemia and high PTH levels were clues for a metabolic bone diasease. Findings of sonography and scintigraphy had hallmarks of a parathyroid pathology. In the light of these evidence, diagnosis of brown tumor in association with PHPT was more likely.

As a rare and benign cause of osteolytic bone involvement, brown tumor is usually due to PHPT caused by an adenoma. Brown tumor causes increment in osteolytic resorption. Generally it is associated with single bone involvement. Diagnosis is based on clinical, radiologic and biochemical findings. After diagnosis of primary hyperparathyrodism is confirmed by biochemical results, a neck sonography confirms the presence of a parathyroid etiology and parathyroid scintigraphy increases the sensitivity for demonstration of a parathyroid pathology [6-7].

After the diagnosis is confirmed, key treatment of brown tumor is surgical removal of parathyroid adenoma. As PTH levels normalize after parathyroid surgery, brown tumor is expected to improve in size or to completely resolve [8-11]. In addition, orthopedic intervention can be necessary in case of pathologic fracture or extensive cortical involvement.

This report emphasizes the value of accurate diagnosis in cases with osteolytic lesions. The possible diagnoses such as metastatic bone involvement, multiple myeloma and metabolic bone diseases such as hyperparathyroid- 
ism due to adenoma have completely different means of treatment including chemotherapy or surgical removal of adenoma. Herein we have reported a case which was, at first glance, very similar to metastatic bone involvement but after a careful examination, it was found to have rather a benign cause. So for avoiding unnecessary interventions brown tumor should be kept in mind for differential diagnosis of osteolytic bone lesions.

\section{ACKNOWLEDGEMENTS}

Informed consent was obtained from the patient.

\section{REFERENCES}

[1] Bruder, J.M. and Mundy, G.R. (2001) Mineral metabolism. In: Felig, P. and Frohman, L.A., Eds., Endocrinology \& Metabolism, 4th Edition, McGraw-Hill, Inc., New York, 1079-1177.

[2] Khalil, P.N., Heining, S.M., Huss, R., Ihrler, S., Siebeck, M., Hallfeldt, K., Euler, E. and Mutschler, W. (2007) Natural history and surgical treatment of brown tumor le- sions at various sites in refractory primary hyperparathy- roidism. European Journal of Medical Research, 12, 222- 230.

[3] Alcalay, M., Azais, I., Brigeon, B., Babin, P., Vandermarcq, P., Debiais, F. and Bontoux, D. (1995) Strategy for identifying primary malignancies with inaugural bone metastases. Revue de Rhumatisme, 62, 632-642.

[4] Brage, M.E. and Simon, M.A. (1992) Evaluation, prognosis, and medical treatment considerations of metastatic bone tumors. Orthopedics, 15, 589-596.
[5] Rougraff, B.T., Kneisl, J.S. and Simon, M.A. (1993) Skeletal metastases of unknown origin. The Journal of Bone \& Joint Surgery, 75, 1276-1281.

[6] Taillefer, R., Boucher, Y., Potvin, C. and Lambert, R. (1992) Detection and localization of parathyroid adenomas in patients with hyperparathyroidism using a single radionuclide imaging procedure with technetinum-99 m-sestamibi (double-phasestudy). Journal of Nuclear Medicine, 33, 1801-1807.

[7] Weiss, R.R., Schoeneman, M.J., Primack, W., Rozycki, D., Bennett, B. and Greifer, I. (1980) Maxillary brown tumor of secondary hyperparathyroidism in a hemodialysis patient. Journal of the American Medical Association, 243, 1929-1930.

[8] Dusunsel, R., Guney, E., de Gunduz, Z., Poyrazoglu, M.H., Yigitbasi, O.G. and Kontas, O. (2000) Maxillary brown tumor caused by secondary hyperparathyroidism in a boy. Pediatric Nephrology, 14, 529-530

[9] Schweitzer, V.G., Thompson, N.W. and McClatchey, K.D. (1986) Sphenoid sinus brown tumor, hypercalcemia, and blindness: An unusual presentation of primary hyperparathyroidism. Head and Neck Surgery, 8, 379-386. doi:10.1002/hed.2890080509

[10] Vander Walde, L.H., Liu, I.L., O’Connell, T.X. and Haigh, P.I. (2006). The effect of parathyroidectomy on bone fracture risk in patients with primary hyperparathyroidism. Archives of Surgery, 141, 885-891. doi:10.1001/archsurg.141.9.885

[11] Simon, M.A. and Springfield, D. (1998) Surgery for bone and soft tissue tumors. Lippincott-Raven, Philadelphia. 